\title{
Lag Secure Consensus for Second-Order Nonlinear Multiagent Systems with Event-Triggered Control Strategy under DoS Attacks
}

\author{
Qi Han $\mathbb{D}^{\mathbb{D}},{ }^{1}$ Ao Zhang $\mathbb{D}^{1},{ }^{1}$ Tengfei Weng, ${ }^{1}$ Yan Xie, ${ }^{1}$ Liping Feng $\mathbb{D},{ }^{2}$ Guorong Chen, ${ }^{1}$ \\ and Yuan Tian ${ }^{1}$ \\ ${ }^{1}$ School of Intelligent Technology and Engineering, Chongqing University of Science and Technology, Chongqing 401331, China \\ ${ }^{2}$ Computer Science Department, Xinzhou Teachers University, Xinzhou, Shanxi 034000, China
}

Correspondence should be addressed to Ao Zhang; zhangaocq@163.com

Received 10 November 2021; Accepted 31 December 2021; Published 19 January 2022

Academic Editor: Chenquan Gan

Copyright (C 2022 Qi Han et al. This is an open access article distributed under the Creative Commons Attribution License, which permits unrestricted use, distribution, and reproduction in any medium, provided the original work is properly cited.

Compared with previous studies, this paper focuses on the time delay phenomenon in the consensus state between the leader and followers and considers that the DoS attack occurs nonperiodically. First, a new event-triggered mechanism and lag consensus control strategy are proposed. Then, through the Lyapunov stability theory, algebraic knowledge, and graph theory, it is proved that followers and leader can achieve lag consistent under the DoS attack, and the trigger interval is designed to ensure that Zeno behavior does not occur. Finally, the correctness and effectiveness of the proposed theory and method are verified by numerical simulation.

\section{Introduction}

In recent years, distributed cooperative control of multi-agent systems (MASs) has been widely used in various fields, such as smart grid collaborative control [1], distributed optimal cooperation [2], coordinated defense systems [3], and so on. In cooperative control, the consensus has always been a hot topic in the research of multi-agent systems. Many researchers have studied the consensus of MASs from different perspectives [4-6]. The overall goal of leader-follower consensus is to drive the states of all followers in the network to track the state of the leader [7]. However, in the actual communication process, many factors, such as the limitation of communication speed, the limited bandwidth, and the asymmetry of information transmission, may lead to delay in transmitting and receiving information between agents [8]. At present, there are many research results on the consensus of communication delay systems. Reference [9] investigated the consensus problem of continuous-time nonlinear MASs with time-varying communication delay via reliable control. The tracking consensus regulation was studied in [10], where high-order MASs was subjected to Lipschitz nonlinear perturbations.
In order to solve the problem of limited resources to a certain extent, this paper introduces an event trigger mechanism to design a control protocol [11]. The eventtrigger mechanism avoids the problem of continuous update of the controller, which not only reduces the amount of data in network transmission but also extends the service life of network components. The event-triggered distributed predictive control (DPC) problem was considered in [12], where multi-agent systems were subject to bounded disturbances. Reference [13] studied the event-triggered containment control problem for a class of networked nonlinear MASs subjected to limited communication resources and proposed a distributed containment output feedback control strategy with an event-triggered communication mechanism.

With the increasingly mature communication technology, the denial-of-service attacks always threaten the normal work of the network systems. The DoS attacks prevent legitimate users from accessing resources properly and even collapse the resources [14]. Therefore, in order to avoid DoS attacks causing damage to the system, some security control protocols have been proposed [15-18]. Reference [19] investigated the secure consensus for second-order MASs with 
nonlinear dynamics and event-triggered control strategy under DoS attacks. In [20], a distributed stochastic model predictive controller was designed for a networked control system with stochastic disturbances and denial-of-service (DoS) attacks. Reference [21] investigated the leader-follower robust $H_{\infty}$ consensus of heterogeneous multiagent systems with denial-of-service attacks. Reference [22] studied the secure consensus problem of multiagent systems under switching topologies, and the studied multiagent systems were affected by both denial-of-service (DoS) attacks and external disturbances. The observer-based output feedback control problem was studied in [23] for cyberphysical systems against randomly occurring packet dropout and periodic DoS attacks. The problem of event-triggered distributed state estimation was considered in [24] for linear multiagent systems under DoS attacks. The distributed event-triggered consensus problem of a generally linear multiagent system was considered in [25] with periodic DoS jamming attacks. Reference [26] applied an input-based triggering approach to investigate the secure consensus problem in multiagent systems under DoS attacks. Reference [27] studied secure L-F consensus of linear MASs under a directed communication network with denial-of-service (DoS) attacks. However, there are few studies on the consensus of nonlinear systems under DoS attacks. In practical applications, most systems are nonlinear, so it is necessary to study nonlinear systems and have practical application value. Compared with the above methods, the distributed control algorithm proposed in this paper achieves the lag consensus of MASs under DoS attacks.

The consensus considered in most of the above literatures was completely consistent $\lim \left\|x_{i}(t)-x_{0}(t)\right\|=0$. However, in the real network, there is a time lag between the state of leader (indexed the leader agent as node 0) and followers, where lag consensus can be denoted $\lim \left\|x_{i}(t)-x_{0}(t-\tau)\right\|=0(\tau>0$ is time delay). In real life, being completely consistent may bring us trouble, and lag consistent can avoid such trouble. For example, many cars are on a road. If they need to reach a certain location at the same time, they may cause a traffic jam. But if they have an appropriate delay on the arrival time, they can pass smoothly and orderly. Reference [28] studied lag consensus of second-order nonlinear MASs. Reference [29] proposed a new distributed controller to solve the prescribed-time cluster lag consensus control for MASs. Reference [30] investigated the problem of cluster lag consensus for firstorder MASs which can be formulated as moving agents in a capacity-limited network. Our three contributions in this paper as follows:

(1) The denial-of-service attacks always threaten the normal work of the network systems. However, there are few studies on the consensus of nonlinear systems under DoS attacks, and the lag consensus in this paper is also rarely studied in this field.

(2) DoS attacks are periodically initiated using known attack strategies in [16], [23], and [25]. However, in practical applications, we often do not predict how DOS attacks will work. Therefore, this paper considers that DoS attacks are nonperiodic and the attack strategy is unknown. In addition, the lag secure consensus of the system is studied in this case.

(3) An event-triggered controller is designed for lag consensus, and the lag secure consensus is studied for second-order nonlinear MASs with event-triggered control strategy under DoS attack. Through the Lyapunov stability method, a sufficient condition is given for ensuring lag secure consensus of multiagent system.

Notations. $\mathbb{R}^{n}$ represents the $n$ - dimensional Euclidean space, the diagonal matrix is represented by $\operatorname{diag}(\cdots) .\|\cdot\|$ refers to the Euclidean norm. $\mathbb{N}$ represents a positive integer. $I_{N n}$ denotes the $N \times n$ identity matrix. The Kronecker product is denoted by $\otimes$. For two sets $Y$ and $Z, Y / Z$ means excluding elements belonging to $Z$ in $Y$.

\section{Problem Formulation}

2.1. Graph Theory. For a multiagent system consisting of $N$ followers, the directed graph $G=(V, E, A)$ represents the communication topology, where $V=\left\{v_{1}, v_{2}, \ldots, v_{N}\right\}$ is the agent set, $E=\left\{e_{j i}=\left(v_{i}, v_{j}\right), v_{i} \neq v_{j}\right\}, \subseteq V \times V$ represents the edge set, and the adjacency matrix of $G$ is represented by $A=\left(a_{i j}\right)_{N \times N} \in \mathbb{R}^{n \times n} .\left(v_{i}, v_{j}\right)$ represents that $v_{j}$ can transmit information to $v_{i}$. If $\left(v_{i}, v_{j}\right) \in E, \forall v_{i}, v_{j} \in V$, then $a_{i j}>0$, and $a_{i j}=0$ otherwise. $D=\operatorname{diag}\left(d_{1}, d_{2}, \ldots, d_{N}\right)$ is a diagonal matrix, where $d_{i}>0$ indicates that the leader can transmit information to node $v_{i}$, otherwise $d_{i}=0$. The Laplace matrix in $G$ satisfies the following definition:

$$
l_{i j}=\left\{\begin{array}{l}
\sum_{j \in N, j \neq i} a_{i j}, \quad i=j, \\
-a_{i j}, \quad i \neq j .
\end{array}\right.
$$

$\bar{G}$ represents the directed graph of the MASs composed of $N$ followers and a leader. Clearly, $G$ is a subgraph of $\bar{G}$.

2.2. System Model. Considering that the MASs in this paper consists of $\mathrm{N}$ followers and a leader, the dynamics of the leader agent is given as

$$
\left\{\begin{array}{l}
\dot{x}_{0}(t)=v_{0}(t) \\
\dot{v}_{0}(t)=g\left(t, x_{0}(t), v_{0}(t)\right)
\end{array}\right.
$$

where $x_{0}(t) \in \mathbb{R}^{n}$ and $v_{0}(t) \in \mathbb{R}^{n}$ represent the position and velocity of the leader respectively, and $g\left(t, x_{0}(t), v_{0}(t)\right) \in \mathbb{R}^{n}$ is a nonlinear function.

The dynamics of the $i$ th follower agent can be described as

$$
\left\{\begin{array}{l}
\dot{x}_{i}(t)=v_{i}(t) \\
\dot{v}_{i}(t)=g\left(t, x_{i}(t)\right), v_{i}(t)+u_{i}(t)
\end{array}\right.
$$

where $i=1,2, \ldots, N, x_{i}(t) \in \mathbb{R}^{n}$ is the position of the $i$ th agent, the velocity of the $i$ th agent can be expressed by $v_{i}(t) \in \mathbb{R}^{n}$, and $u_{i}(t) \in \mathbb{R}^{n}$ represents the control input of the ith agent. $g\left(t, x_{i}(t), v_{i}(t)\right)=\left[g_{1}\left(t, x_{1}(t), v_{1}(t)\right)\right.$, 
$\left.g_{2}\left(t, x_{2}(t), v_{2}(t)\right), \ldots, g_{N}\left(t, x_{i}(t), v_{i}(t)\right)\right]^{T} \in \mathbb{R}^{n}$ is a nonlinear function.

Assumption 1. There exist two nonnegative constants $\beta_{1}$ and $\beta_{2}$, so that $g\left(t, x_{i}(t), v_{i}(t)\right)$ satisfies the following condition:

$$
\begin{array}{r}
\|g(t, x, v)-g(t, y, w)\| \leq \beta_{1}\|x-y\|+\beta_{2}\|v-w\|, \\
\forall x, y, v, w \in \mathbb{R}^{n}, t \in[0, \infty) .
\end{array}
$$

Assumption 2. Assume that the graph $\bar{G}$ is directed and at least one directed spanning tree exists.

2.3. DoS Attack Model. The DoS attack [16-19] refers to intentionally attacking network protocols or a large number of illegal users who directly exhaust the resources of the attacked object. In this paper, DOS attacks are assumed to occur nonperiodic based on time series, and their attack energy is limited. The end of an attack requires stopping to add energy. DoS attacks can attack targets in many ways and its attack strategy is unknown. DoS attacks can affect the control channel so that the agent loses control. Let $\left\{\tilde{t}_{m}\right\}_{m \in \mathbb{N}}$ represents the attack sequence initiated by the denial of service attack at $\widetilde{t}_{m} . \mathrm{H}_{m}=\left[\widetilde{t}_{m}, \widetilde{t}_{m}+\widetilde{\Delta}_{m}\right]$ represents the $m$ th DoS time interval, where $\widetilde{\Delta}_{m}>0$ is length. Obviously, $\tilde{t}_{m+1}>\widetilde{t}_{m}+\widetilde{\Delta}_{m}$. For the given $t>t_{0} \in \mathbb{R}$, similar to [27], let

$$
\prod_{a}\left(t_{0}, t\right)=\cup \mathrm{H}_{m} \cap\left[t_{0}, t\right], \quad m \in \mathbb{N},
$$

denote the time instants set of communication rejected. $\prod_{s}\left(t_{0}, t\right)=\left[t_{0}, t\right] / \prod_{a}\left(t_{0}, t\right)$ represent the sets of time for communication areas.
Definition 1 (secure leader-following lag consensus [8]). A control law $u_{i}(t)$ can achieve the secure lag consensus for MASs (1) and (2) under DoS attacks if the following conditions are satisfied:

$$
\begin{aligned}
& \lim _{t \longrightarrow \infty}\left\|x_{i}(t)-x_{0}(t-\tau)\right\|=0, \quad i=1,2, \ldots, N, \\
& \lim _{t \longrightarrow \infty}\left\|v_{i}(t)-v_{0}(t-\tau)\right\|=0, \quad i=1,2, \ldots, N .
\end{aligned}
$$

For any initial condition $x_{i}(0)$ and $v_{i}(0), i=1,2, \ldots, N$.

Definition 2 (attack frequency [27]). For any $T_{2}>T_{1} \geq t_{0}$, the total number of DoS attacks occurring over $\left[T_{1}, T_{2}\right)$ is denoted by $\mathrm{N}_{a}\left(T_{1}, T_{2}\right)$. Thus, the attack frequency is defined as

$$
F_{a}\left(T_{1}, T_{2}\right)=\frac{\mathrm{N}_{a}\left(T_{1}, T_{2}\right)}{T_{2}-T_{1}}
$$

Definition 3 (attack length rate [19]). For any $t>t_{0}>0$, the total time interval of DoS attacks occurring over $\left[t_{0}, t\right)$ is denoted by $\prod_{a}\left(t_{0}, t\right)$. Thus, the attack length rate is defined as

$$
r_{a}=\frac{\prod_{a}\left(t_{0}, t\right)}{t-t_{0}}
$$

\section{Main Result}

3.1. Event-Triggered Control Scheme Design. Let $t_{0}=t_{0}^{i}, t_{1}^{i}, \ldots, t_{k_{i}}^{i}, \ldots$ represent the event trigger times for agent $i$. An event-triggered control protocol under the DoS attacks was given as follows:

$$
u_{i}(t)=-\varepsilon Z_{i}\left(t_{k_{i}}^{i}\right)=-\varepsilon \sum_{j=1}^{N} a_{i j}\left(x_{i}\left(t_{k_{i}}^{i}\right)-x_{j}\left(t_{k_{j}}^{j}\right)+v_{i}\left(t_{k_{i}}^{i}\right)-v_{j}\left(t_{k_{j}}^{j}\right)\right)-\varepsilon d_{i}\left(x_{i}\left(t_{k_{i}}^{i}\right)-x_{0}\left(t_{k_{i}}^{i}-\tau\right)+v_{i}\left(t_{k_{i}}^{i}\right)-v_{0}\left(t_{k_{i}}^{i}-\tau\right)\right), \quad t \in\left[t_{k_{i}}^{i}, t_{k_{i}+1}^{i}\right),
$$

Where the triggering instant of agent $j$ is represented by $t_{k_{j}}$, and $\varepsilon>0$, and $\tau$ represent the control gain and a time delay, respectively. When $d_{i}>0$, it means that the leader can send information to agent $i$, otherwise $d_{i}=0$. The last successful update is represented by the subscript $k_{i}(t)$, which is described in [27] as follows:

$$
k_{i}(t)=\left\{-1, \quad \text { if } \prod_{s}(0, t) \in \varnothing, \sup \left\{k_{i} \in \mathbb{N} \mid t_{k_{i}}^{i} \in \prod_{s}(0, t)\right\}, \quad\right. \text { otherwise. }
$$

Let

$$
\mathrm{Z}_{i}(t)=\sum_{j=1}^{N} a_{i j}\left(x_{i}(t)-x_{j}(t)+v_{i}(t)-v_{j}(t)\right)+d_{i}\left(x_{i}(t)-x_{0}(t-\tau)+v_{i}(t)-v_{0}(t-\tau)\right)
$$


The Event-trigger error is defined as

$$
\theta_{i}(t)=Z_{i}\left(t_{k_{i}}^{i}\right)-Z_{i}(t)
$$

The Event-trigger function is defined as follows:

$$
f_{i}(t)=\left\|\theta_{i}(t)\right\|-\alpha\left\|Z_{i}\left(t_{k_{i}}^{i}\right)\right\|, \quad \alpha>0 .
$$

The Event-trigger instant sequence of agent $i$ is expressed as

$$
t_{k_{i}+1}^{i}=\inf \left\{t \mid t>t_{k_{i}}^{i}: f_{i}(t) \geq 0\right\}
$$

In order to ensure that the agent does not have Zeno behavior, we adopt the following approach proposed in [31] to determine the event time instant of agent $i$ :

$$
\begin{aligned}
t_{k_{i}+1}^{i} & =t_{k_{i}}^{i}+\widetilde{\Delta}_{k_{i}}^{i}, \\
\widetilde{\Delta}_{k_{i}}^{i} & =\max \left\{\widetilde{\tau}_{k_{i}}^{i}, \widetilde{b}_{i}\right\},
\end{aligned}
$$

where $\widetilde{\Delta}_{k_{i}}^{i}$ represents event interval time, $\widetilde{b}_{i}>0$, and $\widetilde{\tau}_{k_{i}}^{i}$ is defined as follows:

$$
\tilde{\tau}_{k_{i}}^{i}=\inf _{t>t_{k_{i}}^{i}}\left\{t-t_{k_{i}}^{i} \mid f_{i}(t)=0\right\}
$$

3.2. Lag Consensus Analysis. The consensus error is given by

$$
\begin{aligned}
& \widehat{x}_{i}(t)=x_{i}(t)-x_{0}(t-\tau), \\
& \widehat{v}_{i}(t)=v_{i}(t)-v_{0}(t-\tau) .
\end{aligned}
$$

Combining with (9) and (12), systems (1) and (2) can be rewritten as

$$
\left\{\begin{array}{l}
\dot{\hat{x}}_{i}(t)=\widehat{v}_{i}(t), \\
\dot{\widehat{v}}_{i}(t)=\varepsilon \theta_{i}(t)-\varepsilon \sum_{j=1}^{N} a_{i j}\left(\widehat{x}_{j}(t)+\widehat{v}_{j}(t)\right), \\
-\varepsilon d_{i}\left(\widehat{x}_{i}(t)+\widehat{v}_{i}(t)\right)+g\left(t, x_{i}(t), v_{i}(t)\right)-g\left(t, x_{0}(t-\tau), v_{0}(t-\tau)\right) .
\end{array}\right.
$$

Le

t

$$
\begin{aligned}
\widehat{\eta}(t) & =\left[\widehat{x}^{T}(t), \widehat{v}^{T}(t)\right]^{T}, \\
\widehat{x}(t) & =\left[\widehat{x}_{1}(t), \widehat{x}_{2}(t), \ldots, \widehat{x}_{N}(t)\right], \\
\widehat{v}(t) & =\left[\widehat{v}_{1}(t), \widehat{v}_{2}(t), \ldots, \widehat{v}_{N}(t)\right], \\
G(t) & =\left[G^{T}\left(t, \widehat{x}_{1}(t), \widehat{v}_{1}(t)\right), \quad G^{T}\left(t, \widehat{x}_{2}(t), \widehat{v}_{2}(t)\right), \ldots, G^{T}\left(t, \widehat{x}_{N}(t), \widehat{v}_{N}(t)\right)\right]^{T}, \\
G\left(t, \widehat{x}_{i}(t), \widehat{v}_{i}(t)\right) & =g\left(t, x_{i}(t), v_{i}(t)\right)-g\left(t, x_{0}(t-\tau), v_{0}(t-\tau)\right) . \\
\text { 8) as follows: } & \left\|\theta_{i}(t)\right\| \leq \alpha\left\|\mathrm{Z}_{i}\left(t_{k_{i}}^{i}\right)\right\| \leq \alpha\left\|\theta_{i}(t)+\mathrm{Z}_{i}(t)\right\| \leq \alpha\left\|\theta_{i}(t)\right\|+\alpha\left\|\mathrm{Z}_{i}(t)\right\| .
\end{aligned}
$$

Rewriting (18) as follows:

$\dot{\hat{\eta}}(t)=\left[\begin{array}{cc}0 & I_{N} \\ -\varepsilon(L+D) & -\varepsilon(L+D)\end{array}\right] \otimes I_{n} \hat{\eta}(t)+\left[\begin{array}{l}0 \\ \varepsilon\end{array}\right] \otimes I_{n} \theta(t)+\left[\begin{array}{c}0 \\ G(t)\end{array}\right]$.

From $0 \leq \alpha<1 / 3$, we have

It is noticed that

$$
\begin{aligned}
\left\|\theta_{i}(t)\right\| & \leq \frac{\alpha}{1-\alpha}\left\|\mathrm{Z}_{i}(t)\right\|=\frac{\alpha}{1-\alpha}\left\|\sum_{j=1}^{N} a_{i j}\left(x_{i}(t)-x_{j}(t)+v_{i}(t)-v_{j}(t)\right)+d_{i}\left(x_{i}(t)-x_{0}(t-\tau)+v_{i}(t)-v_{0}(t-\tau)\right)\right\| \\
& =\frac{\alpha}{1-\alpha}\left\|\sum_{j=1}^{N} a_{i j}\left(\widehat{x}_{j}(t)+\widehat{v}_{j}(t)\right)+d_{i}\left(\widehat{x}_{i}(t)+\widehat{v}_{i}(t)\right)\right\|
\end{aligned}
$$


Then, we can get

$$
\|\theta(t)\| \leq \omega\|\widehat{\eta}(t)\|,
$$

where $\omega=\alpha / 1-\alpha \lambda_{\max }(L+D)$.

Theorem 1. Suppose that Assumption 1 and 2 holds. Let $\tilde{\gamma}_{1}>0$ and $\tilde{\gamma}_{2}>0$ satisfy $\tilde{\gamma}_{1}+\widetilde{\gamma}_{2}=\tilde{\gamma}<1, \quad \omega^{2} \leq \tilde{\gamma}_{1}$ and $b_{i} \in\left(0,1 / h \ln \left(1+\sqrt{\tilde{\gamma}_{2} / N}\right)\right)$. Lag secure consensus for MASs can be achieved under the following conditions:

(1) There is a constant $\tilde{\eta}_{*} \in\left(0, \xi_{1}\right)$ that makes the attack frequency $F_{a}\left(t_{0}, t\right)$ in Definition 2 satisfy the following inequality:

$$
F_{a}\left(t_{0}, t\right)=\frac{\mathrm{N}_{a}\left(T_{1}, T_{2}\right)}{t-t_{0}} \leq \frac{\tilde{\eta}_{*}}{\ln (\varsigma)+\left(\xi_{1}+\xi_{2}\right) \widetilde{\Delta}_{*}} .
$$

(2) The attack length rate $r_{a}$ in Definition 3 satisfies the following inequality:

$$
r_{a}<\frac{\xi_{1}-\tilde{\eta}_{*}}{\xi_{1}+\xi_{2}}
$$

Proof. Consider two time sequences $\left\{t_{k_{i}}^{i}\right\}_{k_{i} \in \mathbb{N}}$ and $\left\{\tilde{t}_{m}\right\}_{m \in \mathbb{N}}$. The set of attempted updates under DoS attacks is defined as follows:

$$
\Psi=\left\{\left(i, k_{i}\right) \in(V \times \mathbb{N}) \mid t_{k_{i}}^{i} \in \cup_{m \in \mathbb{N}} \mathrm{H}_{m}\right\} .
$$

The agent can recover to a controllable state after the DoS attack, and there must be a time interval $\widetilde{\Delta}_{*}$, which satisfies $\sup _{\left(i, k_{i}\right) \in \Psi} \widetilde{\Delta}_{k_{i}}^{i} \leq \widetilde{\Delta}_{*}$. Obviously, after an attack stops, it takes more than $\widetilde{\Delta}_{*}$ to launch the next attack. The $m$ th time interval is defined as follows:

$$
\phi_{m}=\left[\widetilde{t}_{m}, \tilde{t}_{m}+\widetilde{\Delta}_{m}+\widetilde{\Delta}_{*}\right) .
$$

Two sub-intervals $\widetilde{\Pi}_{a}\left(t_{0}, t\right)$ and $\widetilde{\Pi}_{s}\left(t_{0}, t\right)$ form a time interval $\left[t_{0}, t\right)$, that is

$$
\left[t_{0}, t\right)=\widetilde{\prod_{a}}\left(t_{0}, t\right) \cup \widetilde{\prod_{s}}\left(t_{0}, t\right),
$$

where $\widetilde{\Pi}_{a}\left(t_{0}, t\right)=\cup \phi_{m} \cap\left[t_{0}, t\right]$ and $\widetilde{\Pi}_{s}\left(t_{0}, t\right)=\left[t_{0}, t\right] / \widetilde{\Pi}_{a}$ $\left(t_{0}, t\right)$.

Under the time interval $\widetilde{\prod}_{s}\left(t_{0}, t\right)$, the Lyapunov function is as follows:

$$
\begin{aligned}
V_{S}(t) & =\frac{1}{2} \widehat{\eta}^{T}(t) P \widehat{\eta}(t) \\
& =\frac{1}{2} \widehat{\eta}^{T}(t)\left[\begin{array}{cc}
\varepsilon M \otimes I_{n} & I_{N n} \\
I_{N n} & I_{N n}
\end{array}\right] \widehat{\eta}(t),
\end{aligned}
$$

where $M=(L+D)+(L+D)^{T}>0$.

Taking the time derivatives of $V_{S}(t)$, we have

$$
\begin{aligned}
& \dot{V}_{S}(t)=\widehat{\eta}^{T}(t)\left[\begin{array}{cc}
\varepsilon M \otimes I_{n} & I_{N n} \\
I_{N n} & I_{N n}
\end{array}\right] \dot{\widehat{\eta}}(t) \\
& =\hat{\eta}^{T}(t)\left[\begin{array}{cc}
\varepsilon M \otimes I_{n} & I_{N n} \\
I_{N n} & I_{N n}
\end{array}\right]\left\{\left\{\left[\begin{array}{cc}
0 & I_{N} \\
-\varepsilon(L+D) & -\varepsilon(L+D)
\end{array}\right] \otimes I_{n}\right\} \hat{\eta}(t)+\left[\begin{array}{c}
0 \\
\varepsilon I_{N} \otimes I_{n}
\end{array}\right] \theta(t)+\left[\begin{array}{c}
0 \\
G(t)
\end{array}\right]\right\} \\
& =\widehat{\eta}^{T}(t)\left[\begin{array}{cc}
\varepsilon M \otimes I_{n} & I_{N n} \\
I_{N n} & I_{N n}
\end{array}\right] \times\left[\begin{array}{cc}
0 & I_{N} \otimes I_{n} \\
-\varepsilon(L+D) \otimes I_{n} & -\varepsilon(L+D) \otimes I_{n}
\end{array}\right] \widehat{\eta}(t)+\eta^{T}(t)\left[\begin{array}{c}
\varepsilon I_{N} \otimes I_{n} \\
\varepsilon I_{N} \otimes I_{n}
\end{array}\right] \theta(t)+\widehat{\eta}^{T}(t)\left[\begin{array}{c}
G(t) \\
G(t)
\end{array}\right] \\
& =\frac{1}{2} \widehat{\eta}^{T}(t)\left[\begin{array}{cc}
\varepsilon M & I_{N} \\
I_{N} & I_{N}
\end{array}\right]\left\{\left[\begin{array}{cc}
0 & I_{N} \\
-\varepsilon(L+D) & -\varepsilon(L+D)
\end{array}\right] \otimes I_{n}\right\} \hat{\eta}(t)+\frac{1}{2} \widehat{\eta}^{T}(t)\left\{\left[\begin{array}{cc}
0 & I_{N} \\
-\varepsilon(L+D) & -\varepsilon(L+D)
\end{array}\right] \otimes I_{n}\right\}\left[\begin{array}{c}
\varepsilon M \\
I_{N} \\
I_{N}
\end{array}\right] \widehat{\eta} I_{N}(t) \\
& +\widehat{\eta}^{T}(t)\left[\begin{array}{l}
\varepsilon I_{N} \otimes I_{n} \\
\varepsilon I_{N} \otimes I_{n}
\end{array}\right] \theta(t)+\widehat{\eta}^{T}(t)\left[\begin{array}{l}
G(t) \\
G(t)
\end{array}\right] \\
& =\frac{1}{2} \widehat{\eta}^{T}(t)\left\{\left[\begin{array}{cc}
-\varepsilon M & 0 \\
0 & 2 I_{N}-\varepsilon M
\end{array}\right] \otimes I_{n}\right\} \hat{\eta}(t)+\hat{\eta}^{T}(t)\left[\begin{array}{c}
\varepsilon I_{N} \otimes I_{n} \\
\varepsilon I_{N} \otimes I_{n}
\end{array}\right] \theta(t)+\hat{\eta}^{T}(t)\left[\begin{array}{c}
G(t) \\
G(t)
\end{array}\right] .
\end{aligned}
$$

The last term of equality (30) can be written as 
$\hat{\eta}^{T}(t)\left[\begin{array}{l}G(t) \\ G(t)\end{array}\right]=\sum_{i=1}^{N}\left(\widehat{x}_{i}(t)+\widehat{v}_{i}(t)\right)^{T} G(t) \leq \sum_{i=1}^{N}\left(\left\|\widehat{x}_{i}(t)\right\|+\left\|\hat{v}_{i}(t)\right\|\right)\|G(t)\| \leq \sum_{i=1}^{N}\left(\left\|\widehat{x}_{i}(t)\right\|+\left\|\hat{v}_{i}(t)\right\|\right)\left(\beta_{1}\left\|\hat{x}_{i}(t)\right\|+\beta_{2}\left\|\hat{v}_{i}(t)\right\|\right) \leq \sum_{i=1}^{N} \beta_{1}\left\|\hat{x}_{i}(t)\right\|^{2}$

$$
\begin{aligned}
& +\beta_{2}\left\|\widehat{v}_{i}(t)\right\|^{2}+\frac{\beta_{1}+\beta_{2}}{2}\left(\left\|\widehat{x}_{i}(t)\right\|^{2}+\left\|\widehat{v}_{i}(t)\right\|^{2}\right) \\
= & \frac{3 \beta_{1}+\beta_{2}}{2}\left\|\widehat{x}_{i}(t)\right\|^{2}+\frac{\beta_{1}+3 \beta_{2}}{2}\left\|\widehat{v}_{i}(t)\right\|^{2} \\
= & \frac{3 \beta_{1}+\beta_{2}}{2} \widehat{x}^{T}(t)\left(I_{N} \otimes I_{n}\right) \widehat{x}(t)+\frac{\beta_{1}+3 \beta_{2}}{2} \widehat{v}^{T}(t)\left(I_{N} \otimes I_{n}\right) \hat{v}(t) \\
= & \widehat{\eta}^{T}(t)\left[\begin{array}{cc}
\frac{3 \beta_{1}+\beta_{2}}{2}\left(I_{N} \otimes I_{n}\right) & 0 \\
0 & \frac{\beta_{1}+3 \beta_{2}}{2}\left(I_{N} \otimes I_{n}\right)
\end{array}\right] \widehat{\eta}(t) .
\end{aligned}
$$

The second term of equality (30) can be written as

$$
\begin{aligned}
& \hat{\eta}^{T}(t)\left[\begin{array}{c}
\varepsilon I_{N} \otimes I_{n} \\
\varepsilon I_{N} \otimes I_{n}
\end{array}\right] \theta(t)=\varepsilon \sum_{i=1}^{N}\left(\widehat{x}_{i}(t)+\widehat{v}_{i}(t)\right)^{T} \theta_{i}(t) \leq \varepsilon \sum_{i=1}^{N}\left(\left\|\widehat{x}_{i}(t)\right\|+\left\|\widehat{v}_{i}(t)\right\|\right)\left\|\theta_{i}(t)\right\|, \\
& \widehat{\eta}^{T}(t)\left[\begin{array}{c}
\varepsilon I_{N} \otimes I_{n} \\
\varepsilon I_{N} \otimes I_{n}
\end{array}\right] \theta(t)=\varepsilon \sum_{i=1}^{N}\left(\widehat{x}_{i}(t)+\widehat{v}_{i}(t)\right)^{T} \theta_{i}(t) \leq \varepsilon \sum_{i=1}^{N}\left(\left\|\widehat{x}_{i}(t)\right\|+\left\|\widehat{v}_{i}(t)\right\|\right)\left\|\theta_{i}(t)\right\| .
\end{aligned}
$$

By (22), we hav

$$
\begin{aligned}
& \leq \varepsilon \sum_{i=1}^{N}\left(\left\|\widehat{x}_{i}(t)\right\|+\left\|\widehat{v}_{i}(t)\right\|\right) \times\left(\frac{\alpha}{1-\alpha}\left\|\sum_{j=1}^{N} a_{i j}\left(\widehat{x}_{j}(t)+\widehat{v}_{j}(t)\right)+d_{i}\left(\widehat{x}_{i}(t)+\widehat{v}_{i}(t)\right)\right\|\right) \\
& \leq \frac{\varepsilon \alpha}{1-\alpha}(\widehat{x}(t)+\widehat{v}(t))^{T}\left((L+D) \otimes I_{n}\right)(\widehat{x}(t)+\widehat{v}(t)) \\
& \leq \frac{2 \varepsilon \alpha}{1-\alpha} \widehat{x}(t)^{T}\left((L+D) \otimes I_{n}\right) \widehat{x}(t)+\frac{2 \varepsilon \alpha}{1-\alpha} \widehat{v}(t)^{T}\left((L+D) \otimes I_{n}\right) \hat{v}(t) \\
& \leq \widehat{\eta}^{T}(t)\left[\begin{array}{c}
\frac{2 \varepsilon \alpha}{1-\alpha}\left((L+D) \otimes I_{n}\right) \\
0 \\
\frac{2 \varepsilon \alpha}{1-\alpha}\left((L+D) \otimes I_{n}\right)
\end{array}\right] \widehat{\eta}(t) .
\end{aligned}
$$

Combining (31) and (33), we have

where

$$
\dot{V}_{S}(t) \leq-\frac{1}{2} \widehat{\eta}^{T}(t)\left[\begin{array}{ll}
S & 0 \\
0 & J
\end{array}\right] \widehat{\eta}(t),
$$

$$
S=\frac{\varepsilon(1-3 \alpha)}{1-\alpha}\left(M \otimes I_{n}\right)-\left(3 \beta_{1}+\beta_{2}\right)\left(I_{N} \otimes I_{n}\right) J=\frac{\varepsilon(1-3 \alpha)}{1-\alpha}\left(M \otimes I_{n}\right)-\left(2+\beta_{1}+3 \beta_{2}\right)\left(I_{N} \otimes I_{n}\right) .
$$


Let, $\varepsilon(1-3 \alpha) / 1-\alpha=\gamma, \quad \beta=\max \left\{3 \beta_{1}+\beta_{2} \quad / 2,2+\beta_{1}\right.$

$\left.+3 \beta_{2} / 2\right\}$, we obtain

$$
\begin{aligned}
& \dot{V}_{S}(t) \leq-\frac{1}{2} \widehat{\eta}^{T}(t)\left[\begin{array}{cc}
\gamma\left(M \otimes I_{n}\right)-2 \beta\left(I_{N} \otimes I_{n}\right) & 0 \\
0 & \gamma\left(M \otimes I_{n}\right)-2 \beta\left(I_{N} \otimes I_{n}\right)
\end{array}\right] \hat{\eta}(t) . \\
& M \text {, we have } \gamma\left(M \otimes I_{n}\right)-2 \beta\left(I_{N} \otimes I_{n}\right)
\end{aligned}
$$
$>0$.

When $\gamma>2 \beta / \lambda_{\text {min }}(M)$, we have $\gamma\left(M \otimes I_{n}\right)-2 \beta\left(I_{N} \otimes I_{n}\right)$

Then $\dot{V}_{S}(t) \leq-\xi_{1} V_{S}(t)$, where

$\xi_{1}=\frac{2 \lambda_{\min }(\mathrm{T})}{\lambda_{\max }(P)}$,

Substituting (39), we have

$$
\begin{aligned}
\dot{V}_{a}(t) & =\widehat{\eta}^{T}(t)\left[\begin{array}{cc}
0 & I_{N n} \\
0 & 0
\end{array}\right] \widehat{\eta}(t)+\widehat{\eta}^{T}(t)\left[\begin{array}{c}
0 \\
G(t)
\end{array}\right] \\
& \leq \hat{\eta}^{T}(t)\left[\begin{array}{cc}
\frac{3 \beta_{1}+\beta_{2}}{2} I_{N n} & I_{N n} \\
0 & \frac{\beta_{1}+3 \beta_{2}}{2} I_{N n}
\end{array}\right] \hat{\eta}(t) .
\end{aligned}
$$$$
\mathrm{T}=\left[\begin{array}{cc}
\gamma\left(M \otimes I_{n}\right)-2 \beta\left(I_{N} \otimes I_{n}\right) & 0 \\
0 & \gamma\left(M \otimes I_{n}\right)-2 \beta\left(I_{N} \otimes I_{n}\right)
\end{array}\right] .
$$

We consider system (2) in $\widetilde{\prod}_{a}\left(t_{0}, t\right)$, choose the following Lyapunov function:

$$
V_{a}(t)=\frac{1}{2} \widehat{\eta}^{T}(t) R \widehat{\eta}(t)
$$

where $R=\left[\begin{array}{cc}I_{N n} & 0 \\ 0 & I_{N n}\end{array}\right]$.

We know that when system (2) under DoS attacks, the controller cannot work, that is, $u_{i}(t)=0$. Therefore, $\dot{\hat{\eta}}(t)$ can be written as

$$
\dot{\hat{\eta}}(t)=\left[\begin{array}{cc}
0 & I_{N n} \\
0 & 0
\end{array}\right] \widehat{\eta}(t)+\left[\begin{array}{c}
0 \\
G(t)
\end{array}\right]
$$

Differentiating $V_{a}(t)$, we obtain

It $\begin{gathered}\text { is } \\ \mathrm{K}=\left[\begin{array}{cc}\left(3 \beta_{1}+\beta_{2} / 2\right) I_{N n} & I_{N n} \\ 0 & \left(\beta_{1}+3 \beta_{2} / 2\right) I_{N n}\end{array}\right], \\ \xi_{2}=2 \lambda_{\min }(\mathrm{K}) / \lambda_{\max }(R) .\end{gathered}$ Through $(41)$, we can have
$\dot{V}_{a}(t) \leq \xi_{2} V_{a}(t)$.
$\quad$ It is defined that $V(t)=V_{\sigma(t)}(t)$, where $\sigma(t) \in\{a, b\}$.

It is defined that $V(t)=V_{\sigma(t)}(t)$, where $\sigma(t)$
hrough the Comparison lemma, we can obtain

$V(t) \leq\left\{\begin{array}{l}e^{-\xi_{1}\left(t-\widetilde{t}_{m-1}-\widetilde{\Delta}_{m-1}\right)} V_{a}\left(\widetilde{t}_{m-1}+\widetilde{\Delta}_{m-1}\right), \\ e^{\xi_{2}\left(t-\widetilde{t}_{m}\right)} V_{b}\left(\widetilde{t}_{m}\right) .\end{array}\right.$

If $t \in\left[\widetilde{t}_{m-1}+\widetilde{\Delta}_{m-1}, \widetilde{t}_{m}\right)$, one has

$$
\begin{aligned}
& V(t) \leq e^{-\xi_{1}\left(t-\widetilde{t}_{m-1}-\widetilde{\Delta}_{m-1}\right)} V_{a}\left(\widetilde{t}_{m-1}+\widetilde{\Delta}_{m-1}\right) \leq \varphi e^{-\xi_{1}\left(t-\widetilde{t}_{m-1}-\widetilde{\Delta}_{m-1}\right)} V_{b}\left(\widetilde{t}_{m-1}^{-}+\widetilde{\Delta}^{-}{ }_{m-1}\right) \\
& \leq \varsigma e^{-\xi_{1}\left(t-\tilde{t}_{m-1}-\widetilde{\Delta}_{m-1}\right)}\left[e^{\xi_{2}\left(t-\tilde{t}_{m-2}-\widetilde{\Delta}_{m-2}\right)} \times V_{b}\left(\tilde{t}_{m-2}+\tilde{\Delta}_{m-2}\right)\right] \leq \varsigma^{m} e^{-\xi_{1}}\left|\tilde{\Pi}_{s}\left(t_{0}, t\right)\right| e^{\xi_{2}}\left|\tilde{\Pi}_{a}\left(t_{0}, t\right)\right| V_{a}\left(t_{0}\right) .
\end{aligned}
$$

If $t \in\left[\widetilde{t}_{m}, \widetilde{t}_{m}+\widetilde{\Delta}_{m}+\widetilde{\Delta}_{*}\right)$, one has

$V(t) \leq e^{\xi_{2}\left(t-\tilde{t}_{m}\right)} V_{b}\left(\widetilde{t}_{m}\right) \leq \varsigma^{m+1} e^{-\xi_{1}}\left|\tilde{\Pi}_{s}\left(t_{0}, t\right)\right| e^{\xi_{2}}\left|\tilde{\Pi}_{a}\left(t_{0}, t\right)\right| V_{a}\left(t_{0}\right)$

where $\varsigma=\max \left\{\lambda_{\max }(P) / \lambda_{\text {min }}(R), \lambda_{\text {max }}(R) / \lambda_{\min }(P)\right\}$.

By Definition 2, we can obtain

$$
\mathrm{N}_{a}\left(t_{0}, t\right)=\left\{\begin{array}{l}
m, \quad t \in\left[\widetilde{t}_{m-1}+\widetilde{\Delta}_{m-1}, \widetilde{t}_{m}\right), \\
m+1, \quad t \in\left[\widetilde{t}_{m}, \widetilde{t}_{m}+\widetilde{\Delta}_{m}+\widetilde{\Delta}_{*}\right) .
\end{array}\right.
$$

Therefore, for any $t>t_{0}$, combining (43) and (44), we can obtain

$$
V(t) \leq \varsigma^{N_{a}\left(t_{0}, t\right)} e^{-\xi_{1}\left|\tilde{\Pi}_{s}\left(t_{0}, t\right)\right| e^{\xi_{2}}\left|\tilde{\Pi}_{a}\left(t_{0}, t\right)\right| V\left(t_{0}\right)}
$$

Noticing that

$$
\begin{aligned}
& \left|\widetilde{\prod}_{s}\left(t_{0}, t\right)\right|=t-t_{0}-\left|\widetilde{\prod}_{a}\left(t_{0}, t\right)\right|, \\
& \left|\widetilde{\prod}_{a}\left(t_{0}, t\right)\right| \leq\left|\prod_{a}\left(t_{0}, t\right)\right|+\left(1+N_{a}\left(t_{0}, t\right)\right) \widetilde{\Delta}_{*} .
\end{aligned}
$$

Then combining (47) and (48), we have 


$$
\begin{aligned}
& -\xi_{1}\left|\widetilde{\prod}_{s}\left(t_{0}, t\right)\right|+\xi_{2}\left|\widetilde{\prod}_{a}\left(t_{0}, t\right)\right|=-\xi_{1}\left(t-t_{0}-\left|\widetilde{\prod}_{a}\left(t_{0}, t\right)\right|\right)+\xi_{2}\left|\widetilde{\prod}_{a}\left(t_{0}, t\right)\right|=-\xi_{1}\left(t-t_{0}\right)+\left(\xi_{1}+\xi_{2}\right)\left|\widetilde{\prod}_{a}\left(t_{0}, t\right)\right| \leq-\xi_{1}\left(t-t_{0}\right)+\left(\xi_{1}+\xi_{2}\right) \\
& \quad\left(\left|\prod_{a}\left(t_{0}, t\right)\right|+\left(1+\mathrm{N}_{a}\left(t_{0}, t\right)\right) \widetilde{\Delta}_{*}\right) .
\end{aligned}
$$

By Definition 3, we have

$$
\begin{aligned}
V(t) & \leq \varsigma^{N_{a}\left(t_{0}, t\right)} e^{-\xi_{1}\left(t-t_{0}\right)+\left(\xi_{1}+\xi_{2}\right)\left(\left|\prod_{a}\left(t_{0}, t\right)\right|+\left(1+N_{a}\left(t_{0}, t\right)\right) \tilde{\Delta}_{*}\right)} V\left(t_{0}\right)=e^{N_{a}\left(t_{0}, t\right) \ln \varsigma} e^{-\xi_{1}\left(t-t_{0}\right)+\left(\xi_{1}+\xi_{2}\right)\left(r_{a}\left(t-t_{0}\right)+\left(1+N_{a}\left(t_{0}, t\right)\right) \tilde{\Delta}_{*}\right)} V\left(t_{0}\right) \\
& =e^{\left(\ln \varsigma+\left(\xi_{1}+\xi_{2}\right) \tilde{\Delta}_{*}\right) N_{a}\left(t_{0}, t\right)} e^{\left(-\xi_{1}+r_{a}\left(\xi_{1}+\xi_{2}\right)\right)\left(t-t_{0}\right)} e^{\left(\xi_{1}+\xi_{2}\right) \tilde{\Delta}_{*}} V\left(t_{0}\right)
\end{aligned}
$$

Combining (24) and (25), the previous inequality can be rewritten as

$$
V(t) \leq e^{\tilde{\eta}_{*}\left(t-t_{0}\right)} e^{\left(-\xi_{1}+r_{a}\left(\xi_{1}+\xi_{2}\right)\right)\left(t-t_{0}\right)} e^{\left(\xi_{1}+\xi_{2}\right) \tilde{\Delta}_{*}} V\left(t_{0}\right)=e^{-\left(-\tilde{\eta}_{*}+\xi_{1}-r_{a}\left(\xi_{1}+\xi_{2}\right)\right)\left(t-t_{0}\right)} e^{\left(\xi_{1}+\xi_{2}\right) \tilde{\Delta}_{*}} V\left(t_{0}\right) \leq e^{-\eta_{*}\left(t-t_{0}\right)} e^{\left(\xi_{1}+\xi_{2}\right) \tilde{\Delta}_{*}} V\left(t_{0}\right)
$$

where $\eta_{*}=-\widetilde{\eta}_{*}+\xi_{1}-r_{a}\left(\xi_{1}+\xi_{2}\right)>0$.

Next, we will prove that the proposed control strategy can exclude the Zeno behavior. The Zeno behavior is that in the event trigger control, the control is triggered infinitely in a finite time. The interevent time of the agent is proposed in (15). Let $W_{1}(t)$ represent the set of agents with the latest interevent time of $\widetilde{\tau}_{k_{i}}^{i}$ and $W_{2}(t)$ represent the set of agents with the latest interevent time of $\widetilde{b}$. Let $\widetilde{\gamma}_{1}+\widetilde{\gamma}_{2}=\tilde{\gamma}<1$, we have

$$
\begin{gathered}
\sum_{i \in W_{1}(t)}\left\|\theta_{i}(t)\right\|^{2} \leq \tilde{\gamma}_{1} \sum_{i \in W_{1}(t)}\left\|\widehat{\eta}_{i}(t)\right\|^{2} \leq \tilde{\gamma}_{1} \sum_{i=1}^{N}\left\|\widehat{\eta}_{i}(t)\right\|^{2}, \\
\sum_{i \in W_{2}(t)}\left\|\theta_{i}(t)\right\|^{2} \leq \tilde{\gamma}_{2} \sum_{i \in W_{2}(t)}\left\|\hat{\eta}_{i}(t)\right\|^{2} \leq \tilde{\gamma}_{2} \sum_{i=1}^{N}\left\|\hat{\eta}_{i}(t)\right\|^{2} .
\end{gathered}
$$

For the agent in $W_{1}(t),\|\theta(t)\| \leq \omega\|\widehat{\eta}(t)\|$ with $\omega^{2} \leq \widetilde{\gamma}_{1}$. Considering the agent in $W_{2}(t)$, from (53), we have $\left\|\theta_{i}(t)\right\|^{2} \leq \sum_{j=1}^{N} \widetilde{h}\left\|\widehat{\eta}_{j}(t)\right\|^{2}$, where $\widetilde{h}=\widetilde{\gamma}_{2} / N$. For the agent in $W_{2}(t), t_{k_{i}+1}^{i}=t_{k_{i}}^{i}+\widetilde{b}_{i}$ can ensure (53). To prove that the interevent time is greater than 0 , we have

$$
\frac{\mathrm{d}}{\mathrm{d} t} \frac{\left\|\theta_{i}(t)\right\|}{\|\widehat{\eta}(t)\|} \leq\left(1+\frac{\left\|\theta_{i}(t)\right\|}{\|\widehat{\eta}(t)\|}\right) \frac{\|\dot{\hat{\eta}}(t)\|}{\|\widehat{\eta}(t)\|}
$$

By (39), we have

$$
\frac{\left[\begin{array}{c}
0 \\
G(t)
\end{array}\right]}{\|\hat{\eta}(t)\|} \leq\left\|\begin{array}{lc}
0 & 0 \\
0 & \frac{\beta_{1}+3 \beta_{2}}{2} I_{N n}
\end{array}\right\| .
$$

Combining (20) and (55), we have

$$
\frac{d}{d t} \frac{\left\|\theta_{i}(t)\right\|}{\|\widehat{\eta}(t)\|} \leq h\left(1+\frac{\left\|\theta_{i}(t)\right\|}{\|\widehat{\eta}(t)\|}\right)
$$

where

$h=\left\|\left[\begin{array}{cc}0 & I_{N n} \\ -\varepsilon(L+D) \otimes I_{n} & -\varepsilon(L+D) \otimes I_{n}\end{array}\right]\right\|+\left\|\left[\begin{array}{l}0 \\ \varepsilon\end{array}\right] \otimes I_{n}\right\| \sqrt{\tilde{h}}+$
$\left\|\begin{array}{cc}0 & 0 \\ 0 & \beta_{1}+3 \beta_{2} / 2 I_{N n}\end{array}\right\|$. Therefore, $\widetilde{\mathrm{B}}=1 / h \ln \left(1+\sqrt{\widetilde{\gamma}_{2} / N}\right)$ determines the minimum time for $\left\|\theta_{i}(t)\right\| /\|\widehat{\eta}(t)\|$ to evolve from 0 to $\sqrt{\tilde{h}}$. Obviously, for the agent in $W_{2}(t), \widetilde{b}_{i} \leq \widetilde{\mathrm{B}}$ can be guaranteed (53). It is concluded that the controller (9) based on event trigger (13) guarantees that (51) holds for all agents, which means $\lim V(t)=0$. That is, systems (1) and (2) can achieve the

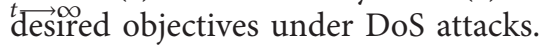

\section{Numerical Simulation}

This section verifies the correctness and effectiveness of the proposed theory and method by an example. We construct a nonlinear second-order system with four followers and one leader, where the communication topology is shown in Figure 1.

$$
\text { By calculation, we have } M=\left[\begin{array}{cccc}
4 & -1 & -1 & -1 \\
-1 & 4 & -1 & -1 \\
-1 & -1 & 2 & -1 \\
-1 & -1 & -1 & 6
\end{array}\right] \text {. }
$$

The initial values of the leader's position and velocity in system (1) are -7 and 1 , respectively. The initial value of the follower is $[3,-2,3,0.3 ; 6,-2,2,1]$. The nonlinear function is $g\left(t, x_{i}(t), v_{i}(t)\right)=0.4 \cos \left(v_{i}(t)\right)+0.1 \sin \left(x_{i}(t)\right)$.

Let $\beta_{1}=\beta_{2}=0.003, \tau=5 s, \varepsilon=3$, and $\tilde{\eta}_{*}=0.02$. By calculation, we have $\xi_{1}=0.043, \quad \xi_{2}=0.012$, $\varsigma=\max \left\{\lambda_{\max }(P) / \lambda_{\min }(R), \lambda_{\max }(R) / \lambda_{\min }(P)\right\}=2.989$, 


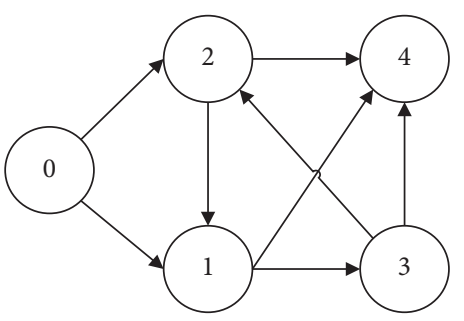

Figure 1: A second-order system topo
adjacency matrix is $A=\left[\begin{array}{lllll}0 & 1 & 0 & 0 \\ 0 & 0 & 1 & 0 \\ 1 & 0 & 0 & 0 \\ 1 & 1 & 1 & 0\end{array}\right]$.

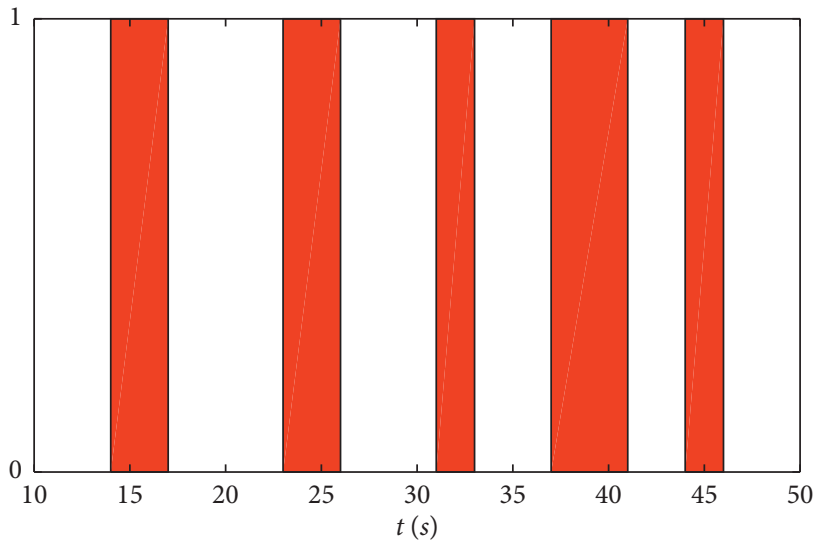

Figure 2: Sequence of DoS attacks.

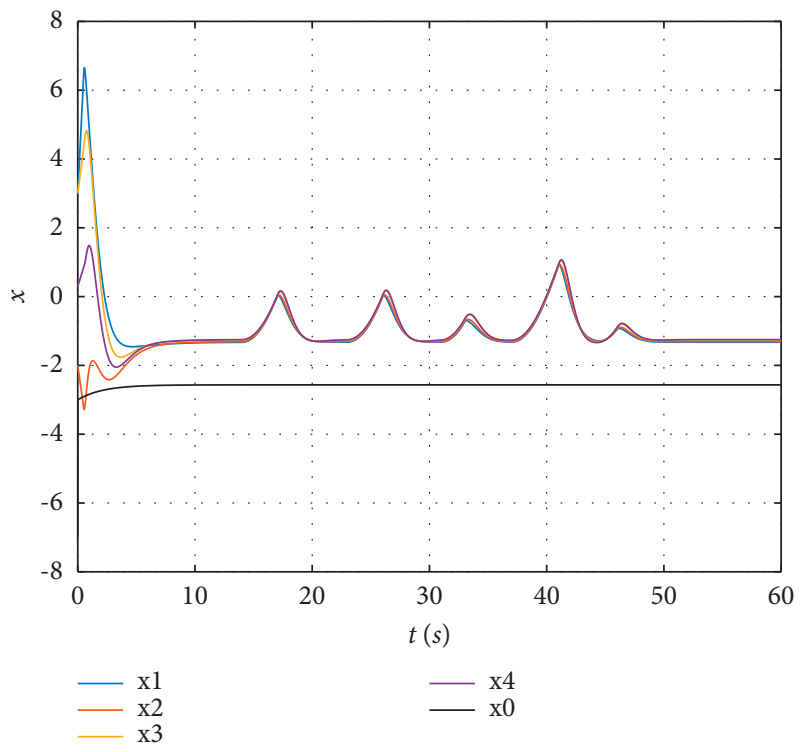

FIGURE 3: The position trajectory of the agent.

$\lambda_{\text {min }}(M)=0.506$, and $\beta=2.012$, so that we can get $\gamma=8.8$, $r_{a}=0.233<\xi_{1}-\tilde{\eta}_{*} / \xi_{1}+\xi_{2}=0.420$, and $F_{a}\left(t_{0}, t\right) \leq 0.003$. Figure 2 shows the sequence of DoS attacks. Figures 3 and 4 show the trajectory of the position and velocity of the five agents, respectively. We can see that there is a delay in the consistent state between the leader and the follower. Therefore, the leader and the follower achieve lag consensus under the DoS attack. Errors of leader-following lag consensus are shown in Figures 5 and 6 . Obviously, the lag error between followers and leader finally tends to 0 , indicating that the multiagent system achieves lag consensus. Figures 5 and 6 show that the control protocol designed in this paper can effectively defend against nonperiodic DoS attacks. Figure 7 shows 


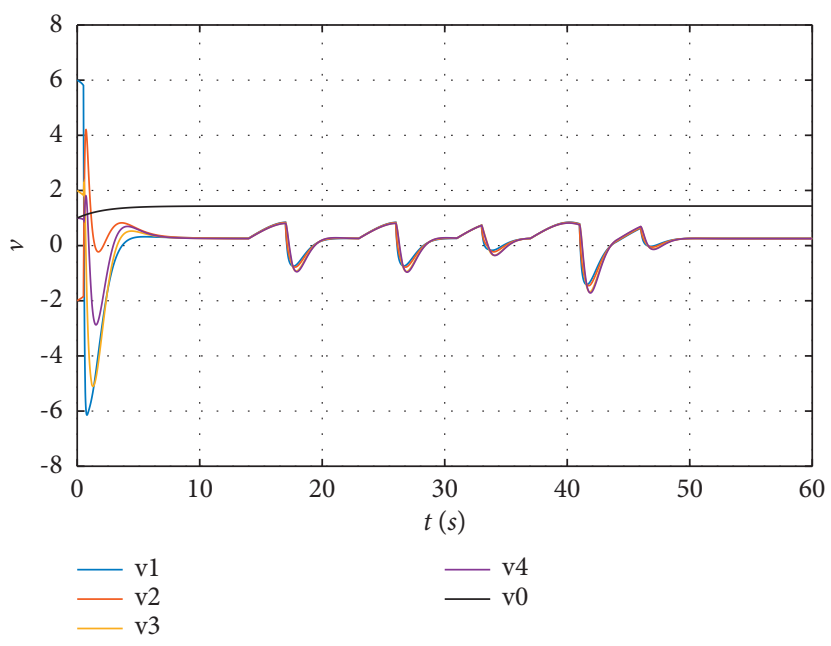

FIgURE 4: Velocity trajectory of agents.

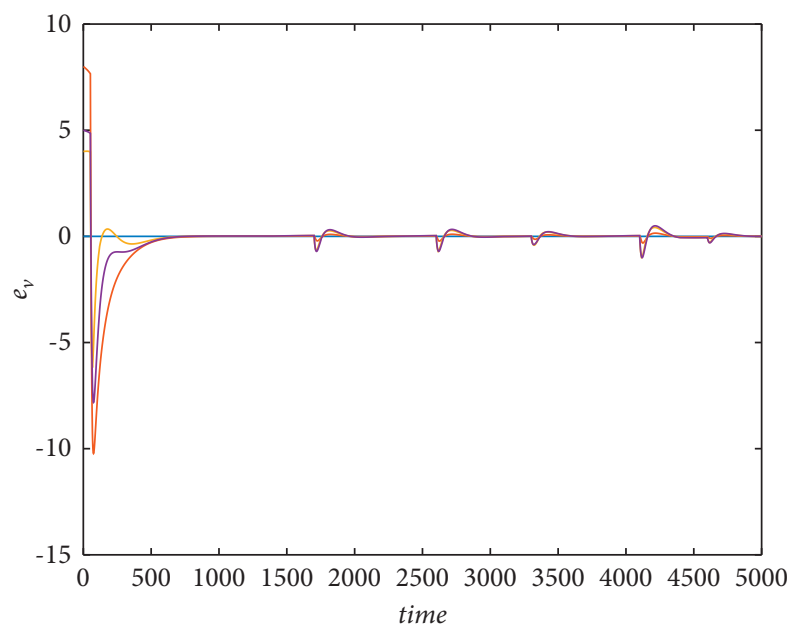

FIGURE 5: Velocity error trajectory of agents.

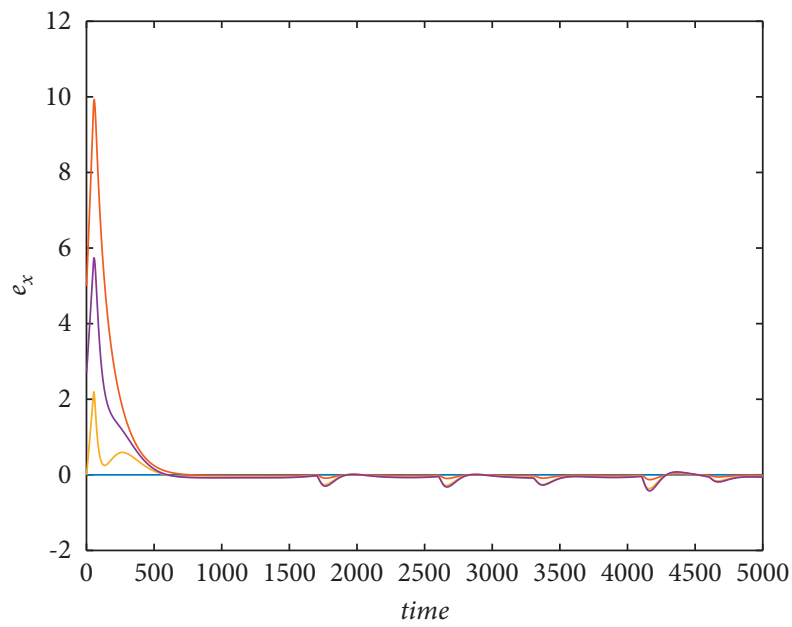

FIgURE 6: Position error trajectory of agents. 


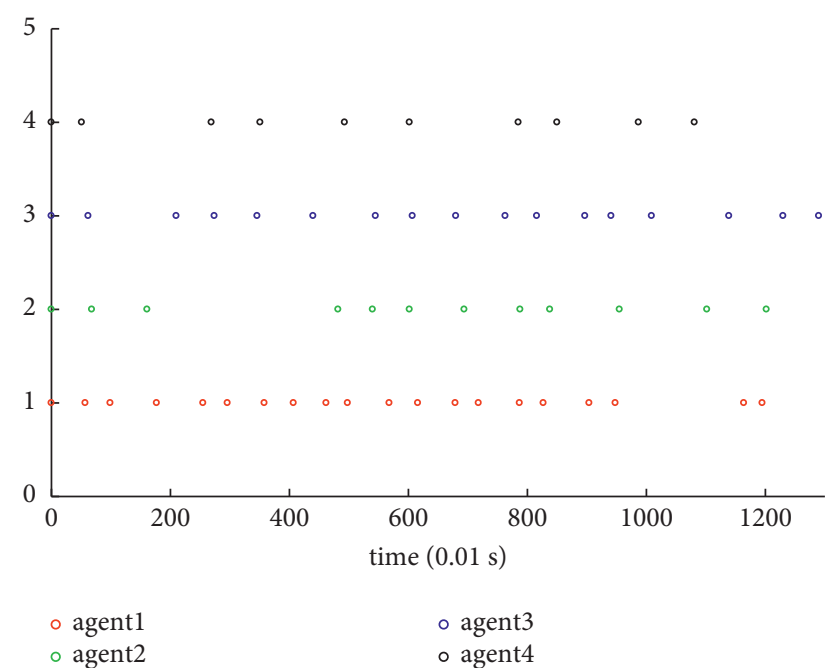

FIgURE 7: Trigger interval.

the event trigger instants for four agents. Obviously, this article can effectively avoid the Zeno behavior by setting the minimum event trigger interval.

\section{Conclusion}

This paper focuses on the time-delay phenomenon in the consensus state of the leader and followers and studies the lag secure consensus problem of MASs under the nonperiodic DoS attack.

In order to achieve the lag consensus between a leader and followers, this paper proposes a security control strategy based on event triggering and derives the upper bound of the frequency and length rate of DoS attacks. Without exceeding the upper bound, the MASs can achieve lagging security consensus. In future works, we will study the high-order multiagent system with communication delay and discuss the lag consensus of MASs under DoS attack.

\section{Data Availability}

The data are available from the corresponding author upon request.

\section{Conflicts of Interest}

The authors declare that they have no conflicts of interest.

\section{Acknowledgments}

This work was supported in part by Humanities and Social Sciences Research of Ministry of Education (19YJCZH047), in part by the West Light Foundation of the Chinese Academy of Science, in part by the Scientific and Technological Research Program of Chongqing Municipal Education Commission (KJZDK201901504and KJQN201901537), in part by the Research Foundation of The Natural Foundation of Chongqing City (cstc2021jcyj-msxmX0146), and in part by the Science and Technology Planning Project of Luzhou City (2021-JYJ-92). The authors would like to thank the support of China Scholarship Council.

\section{References}

[1] B. Li, R. Lu, W. Wang, and K. K. R. Choo, "Distributed hostbased collaborative detection for false data injection attacks in smart grid cyber-physical system," Journal of Parallel and Distributed Computing, vol. 103, pp. 32-41, 2017.

[2] Z. Li, Z. Wu, Z. Li, and Z. Ding, "Distributed optimal coordination for heterogeneous linear multiagent systems with event-triggered mechanisms," IEEE Transactions on Automatic Control, vol. 65, no. 4, pp. 1763-1770, 2020.

[3] B. L. Su and Y. X. Duan, "Distributed optimal control of nonlinear time-delay system subject to delayed measurements and communication disruptions," Journal of Systems Science and Complexity, vol. 34, no. 4, pp. 1426-1437, 2021.

[4] W. Zhu, H. Pu, D. Wang, and H. Li, "Event-based consensus of second-order multi-agent systems with discrete time," Automatica, vol. 79, pp. 78-83, 2017.

[5] W. Zhu, D. D. Wang, and Q. H. Zhou, "Leader-following consensus of multi-agent systems via adaptive event-based control," Journal of Systems Science and Complexity, vol. 32, no. 3, pp. 114-124, 2019.

[6] H. Chu, D. Yue, C. Dou, and L. Chu, "Adaptive PI control for consensus of multiagent systems with relative state saturation constraints," IEEE Transactions on Cybernetics, vol. 54, no. 1, pp. 1-7, 2019.

[7] H. Zhang and F. L. Lewis, "Adaptive cooperative tracking control of higher-order nonlinear systems with unknown dynamics," Automatica, vol. 48, no. 7, pp. 1432-1439, 2012.

[8] Y. Y. Xie, Y. Wang, and Z. J. Ma, "Delay consensus of leaderfollowing multi-agent systems," Acta Physica Sinica, vol. 63, no. 4, Article ID 040202, 2014.

[9] K. Subramanian, P. Muthukumar, and Y. H. Joo, "Leaderfollowing consensus of nonlinear multi-agent systems via reliable control with time-varying communication delay," International Journal of Control, Automation and Systems, vol. 17, pp. 1-9, 2019.

[10] X. L. Jiang, G. H. Xia, Z. G. Feng, and Z. Y. Jiang, "Consensus tracking of data-sampled nonlinear multi-agent systems with packet loss and communication delay," IEEE Transactions on Network Science and Engineering, vol. 8, no. 1, 2020.

[11] S. M. Chen, J. J. Guan, Y. L. Gao, and H. C. Yan, "Observerbased Event-Triggered Tracking Consensus of Non-ideal General Linear Multi-Agent Systems," Journal of the Franklin Institute, vol. 35617, 2018.

[12] Y. Zou, X. Su, S. Li, Y. Niu, and D. Li, "Event-triggered distributed predictive control for asynchronous coordination of multi-agent systems," Automatica, vol. 99, pp. 92-98, 2019.

[13] Y. Yang and Y. Qian, "Event-trigger-based recursive slidingmode dynamic surface containment control with nonlinear gains for nonlinear multi-agent systems," Information Sciences, vol. 560, pp. 202-216, 2021.

[14] M. Cong, X. Mu, and Z. Hu, "Sampled-data-based eventtriggered secure bipartite tracking consensus of linear multiagent systems under DoS attacks," Journal of the Franklin Institute, vol. 358, no. 13, pp. 6798-6817, 2021.

[15] Y. Li, P. Zhang, and L. Ma, "Denial of service attack and defense method on load frequency control system," Journal of the Franklin Institute, vol. 356, no. 15, pp. 8625-8645, 2019. 
[16] S. Liu, S. B. Li, and B. G. Xu, "Event-triggered resilient control for cyber physical system under denial-of-service attacks," International Journal of Control, vol. 93, no. 8, pp. 1-24, 2018.

[17] X. Li, C. Wen, J. Wang, C. Chen, and C. Deng, "Resilient leader tracking for networked Lagrangian systems under DoS attacks," Information Sciences, vol. 577, no. 6, pp. 622-637, 2021.

[18] X. Shao and D. Ye, "Neural-network-based adaptive secure control for nonstrict-feedback nonlinear interconnected systems under DoS attacks," Neurocomputing, vol. 448, pp. 263-275, 2021.

[19] T. Dong and Y. Gong, "Leader-following secure consensus for second-order multi-agent systems with nonlinear dynamics and event-triggered control strategy under DoS attack," Neurocomputing, vol. 416, pp. 95-102, 2019.

[20] H. Yang, Y. Li, L. Dai, and Y. Xia, "MPC-based defense strategy for distributed networked control systems under DoS attacks," Systems \& Control Letters, vol. 128, pp. 9-18, 2019.

[21] D. Zhang and G. Feng, "A new switched system approach to leader-follower consensus of heterogeneous linear multiagent systems with DoS attack," IEEE Transactions on Systems, Man, and Cybernetics: Systems, vol. 51, no. 2, pp. 1-9, 2019.

[22] S. Du, Y. Wang, L. Dong, and X. Li, "Secure consensus of multiagent systems with DoS attacks via a graph-based approach," Information Sciences, vol. 570, pp. 94-104, 2021.

[23] L. Su and D. Ye, "Observer-based output feedback $\mathrm{H} \infty$ control for cyber-physical systems under randomly occurring packet dropout and periodic DoS attacks," ISA Transactions, vol. 104, 2019.

[24] Y. C. Sun and G. H. Yang, "Event-triggered distributed state estimation for multiagent systems under DoS attacks," IEEE Transactions on Cybernetics, pp. 1-10, 2020.

[25] Z. H. Cheng, D. Yue, S. L. Hu, H. Gei, and L. Chen, "Distributed event-triggered consensus of multi-agent systems under periodic DoS jamming attacks," Neurocomputing, vol. 400, pp. 458-466, 2019.

[26] Y. Xu, M. Fang, Z. G. Wu, and Y. J. Pan, "Input-based eventtriggering consensus of multiagent systems under denial-ofservice attacks," IEEE Transactions on Systems, Man, and Cybernetics: Systems, vol. 50, pp. 1-10, 2018.

[27] F. Zhi, G. H. Wen, and G. Q. Hu, "Distributed secure coordinated control for multiagent systems under strategic attacks," IEEE Transactions on Cybernetics, vol. 47, no. 5, pp. 1273-1284, 2017.

[28] Y. Wang and Z. J. Ma, "Lag consensus of the second-order leader-following multi-agent systems with nonlinear dynamics," Neurocomputing, vol. 171, pp. 82-88, 2016.

[29] Y. H. Ren, W. N. Zhou, Z. W. Li, L. Liu, and Y. Q. Sun, "Prescribed-time cluster lag consensus control for secondorder non-linear leader-following multiagent systems," ISA Transactions, vol. 109, no. 6, 2020.

[30] Y. Wang, Z. Ma, and G. Chen, "Distributed control of cluster lag consensus for first-order multi-agent systems on QUAD vector fields," Journal of the Franklin Institute, vol. 355, no. 15, pp. 7335-7353, 2018.

[31] Y. Fan, 1. Liu, G. Feng, and Y. Wang, "Self-triggered consensus for multi-agent systems with Zeno-free triggers," IEEE Transactions on Automatic Control, vol. 60, no. 10, 2015. 